\title{
Breastfeeding duration related to practised contraception in the Netherlands
}

Jacobus P van Wouwe (ko.vanwouwe@tno.nl)' ${ }^{1}$, Caren I Lanting ${ }^{1}$, Paula van Dommelen' ${ }^{1}$, Pieter E Treffers' ${ }^{1}$, Stef van Buuren ${ }^{1,2}$

1.Netherlands Organization for Applied Scientific Research TNO Quality of Life, Leiden, the Netherlands

2.University of Utrecht, Applied Statistics in Prevention, Utrecht, the Netherlands

\section{Keywords}

Breastfeeding duration, Epidemiology, Imputation, Lactation, Practised contraception

\section{Correspondence}

Jacobus P van Wouwe, M.D., Ph.D., Netherlands Organization for Applied Scientific Research TNO,

P.O. Box 2215, 2301 CE Leiden, the Netherlands.

Tel: +31-71-518-1758 |

Fax: +31-71-518-1920।

E-mail: ko.vanwouwe@tno.nl

\section{Received}

17 June 2008; revised 5 August 2008; accepted 8 August 2008.

DOI:10.1111/j.1651-2227.2008.01019.x

\begin{abstract}
Aim: The aim of this study was to gain insight into contraception practised and related to breastfeeding duration.

Methods: Mothers with infants up to 6 months received a questionnaire on infant feeding (breast or formula feeding) and contraception (hormonal or non-hormonal methods). Estimates of the time interval between resuming contraception and cessation of lactation was calculated by Chained Equations Multiple Imputation.

Results: Of all women $(n=2710), 30 \%$ choose condoms, $22 \%$ the combined oral contraceptive pill (OCP) and few other methods. Breastfeeding was started by $80 \%$, and $18 \%$ continued up to 6 months. Of the breastfeeding mothers, $5 \%$ used hormonal contraception; $7 \%$ of women who used hormonal contraception practised breastfeeding. After adjustment for background variables, the use of OCP is strongly associated with formula feeding: after delivery to the third month postpartum, the crude OR being 17.5 (95\% Cl: 11.3-27.0), the adjusted OR 14.5 (9.3-22.5); between the third and sixth month postpartum, respectively, 13.1 (95\% Cl: 8.6-19.9) and 11.7 (7.6-17.9). Of all breastfeeding women, $20-27 \%$ resumed OCP at 25 weeks postpartum and $80 \%$ introduced formula feeding. The time lag between these events is 6 weeks. Hormonal contraception was resumed after formula introduction.

Conclusion: Mothers avoid hormonal contraception during lactation; they change to formula feeding 6 weeks before they resume the OCP. To effectively promote longer duration of breastfeeding, the BFHI needs to address contraception as practised.
\end{abstract}

\section{INTRODUCTION}

The preventive health effects of breastfeeding are clearly acknowledged for developing as well as developed countries (1). The World Health Organization (WHO) recommends exclusive breastfeeding for a minimum of 6 months (2). Despite coordinated actions on national and international level, not many women in the developed world achieve this (3). The subject of adequate promotion of breastfeeding still deserves attention.

The WHO and the International Planned Parenthood Federation consider the use of the combined oral contraceptive pill (OCP) during lactation a risk (4) as 'it hampers milk production and causes insufficient intake in infants'. Yet little evidence is present whether this claim is correct or relevant (5). Experts in the UK and elsewhere could not reach consensus on whether or not to prescribe the OCP during lactation (6-8).

With this study, we want to add to the discussion by providing insight into the current practice and beliefs of lactating mothers regarding their choice of contraception. Until now little is known about this subject, besides a study from Peru performed between 1986 and 1992 (9). We looked into the frequencies of use of various contraceptive methods in a large group of Dutch lactating mothers, who participated in a survey on infant-feeding practices. We also studied the sequence of, and the time interval between, the introduction of formula feeding and the start of OCP. Under the WHO assumption, one expects breastfeeding women to first start OCP and subsequently introduce formula feeds, in order to compensate for the alleged decreased milk production. On the other hand, mothers and midwives express concerns about exposure of infants to contraceptive hormones via human milk. This idea may also be relevant to the current practice. In that case, the sequence of events more likely is reversed, which is first cessation of breastfeeding and introduction of formula feeding, followed by the start of hormonal contraceptives.

\section{METHOD}

\section{Study population and data collection}

About $98 \%$ of Dutch mothers regularly visit a well-baby clinic with their infant. In total 222 well-baby clinics participated in the survey. Health care workers handed out questionnaires on infant feeding and contraceptive behaviour to the first 20 mothers with infants up to 6 months of age visiting the centre at a predetermined week during the year 2002. Mothers were asked to fill out the questionnaire at home and send it back anonymously in a pre-paid envelope.

The questionnaire contained the questions: 'Do you practise contraception? When did you start this (in weeks after delivery)? What method did you use?' The options given were OCP, injectable, IUD, condom or other (non-specified) 
method. Brand names of the contraceptive OCP were used to identify compositions. Non-hormonal contraception was defined as use of condom or IUD, and hormonal contraception as OCP's or injectables. Also mothers were asked whether they fed their infant on breast milk, on combined feeds (breast milk and formula milk in various proportions) or on formula milk. Breastfeeding mothers may occasionally supply a drink of water and, to infants aged 4 months or over, some solids. Separate questions were asked when mothers introduced formula milk and when they ceased to breastfeed.

Several background variables were recorded, including maternal age (in years), parental level of formal education (low, intermediate or high), current smoking habit (no/yes) and mother's employment status before pregnancy (hours per week). From the literature, these variables are known to be related to the type of infant feeding. More detailed information on all the data collected by the questionnaire is provided elsewhere (3).

\section{Data analyses}

We started with producing contingency tables of types of feeding against users of different classes of contraception (hormonal or non-hormonal). Comparison between groups was expressed as crude and adjusted odds ratios with 95\% confidence intervals. Adjustments were made for the aforementioned background variables.

After that, in order to be able to relate resumption of contraception to introduction of formula feeding, we had to obtain both moments for all individuals. However, as mentioned earlier, the questionnaire was handed out to mothers up to 26 weeks after delivery. For mothers with the youngest infants one or both of the events may not have yet occurred. The timing of the events is only known up to the moment that the questionnaire was completed; in other words, these are censored data. Failing to account for the censoring leads to biased estimates of the time interval between both events. Standard survival regression techniques however are limited to one outcome measure, and cannot be used. As an alternative, we applied multiple imputation that yields uncensored event times, which we could have observed if we had run an experiment long enough so that all mothers had introduced formula feeding and resumed contraception (10). We used Multiple Imputation by Chained Equations (MICE) (11). We specified the imputation model for introduction of formula conditional on the current use of contraception (hormonal or non-hormonal). In contrast, the imputation model for resumption of contraception was conditional on current feeding (formula or exclusively breast). The MICE algorithm yields imputations for each event thereby taking both imputation models into account. The resulting data set is the imputed data. Differences between groups were considered significant if $\mathrm{p}<0.05$.

\section{RESULTS}

We obtained responses from 2874 mothers. They had visited during the predetermined week 1 of the 222 participat-
Table 1 Selected maternal characteristics $(n=2710)$

\begin{tabular}{lll} 
Maternal characteristics & Study group & Nationwide' $^{1}$ \\
\hline $\begin{array}{l}\text { Age (years) } \\
\text { Mean ( } \pm \text { SD, range) }\end{array}$ & $31(4,15-46)$ & 31.2 \\
Origin by birth, n (\%) & & \\
$\quad$ Dutch & $2.545(94.3)$ & 91.2 \\
$\quad$ Non-Dutch & $155(5.7)$ & 9.8 \\
Educational level, n (\%) & & \\
Low & $303(11.2)$ & 25.6 \\
Intermediate & $1094(40.3)$ & 45.9 \\
High & $1289(47.6)$ & 28.2 \\
Unknown & $24(0.9)$ & 0.3 \\
Current smoking, n (\%) & & \\
$\quad$ No & $2303(85.0)$ & 85 \\
Yes & $398(14.7)$ & $15^{2}$ \\
Unknown & $9(0.3)$ & \\
Employment status before pregnancy, n (\%) & & \\
$\quad \geq 32$ h/week & $1128(41.6)$ & 34.8 \\
16-31 h/week & $922(34.0)$ & 38.6 \\
$\quad<16$ h/week or no job & $656(24.2)$ & 26.5 \\
Unknown & $4(0.1)$ & 0.1 \\
\hline
\end{tabular}

${ }^{1}$ Data by statistics Netherlands, www.cbs.nl.

${ }^{2}$ Data by www.stivoro.nl.

ing well-baby clinics situated in neighbourhoods of variable social status throughout the country. The responses were equally distributed from all over the Netherlands. Table 1 presents some characteristics of the study group. Women with lower educational level are underrepresented in comparison to the general population of women aged between 20 and 40 years living in the Netherlands. The analyses were restricted to 2710 mothers who answered all questions on infant feeding and the use of contraception.

In the study group, the most commonly used nonhormonal contraceptive was the condom $(30 \%)$. The levonorgestrel-loaded IUD was not popular $(<<1 \%)$; IUD's in general were used by $5 \%$ of women. The use of other nonhormonal methods was reported by $2 \%$ of women (mainly sterilization of woman or partner). By far the most popular hormonal contraceptive was the OCP containing $30 \mu \mathrm{g}$ ethinyl estradiol (it was used by $22 \%$ of all subjects). The following methods were not often used: OCP with $50 \mu \mathrm{g}$ ethinyl estradiol $(<1 \%)$, the injectable progestin-only $(<1 \%)$ and the mini-pill (progestin-only, 2\%). Many women $(80 \%)$ started breastfeeding. At the end of the 6 -month period about $18 \%$ continued exclusive breastfeeding.

Table 2 provides the frequency distribution of infant feeding and practised contraception during the first and second 3-month period after birth. There is a strong association between type of infant feeding and type of contraception in both periods after delivery. In the 6-month period after delivery, the use of OCP is strongly associated with formula feeding. In the first 3-month period, the crude OR is 17.5 (95\% CI: 11.3-27.0) and after adjustment for background variables (maternal age, education, smoking and employment 
Table 2 Breast or formula feeding and non-hormonal or hormonal contraception during 6 months postpartum ( $n=2710$, combined feeding not shown)

\begin{tabular}{|c|c|c|c|c|}
\hline \multirow[t]{2}{*}{ Contraception } & \multirow{2}{*}{$\begin{array}{l}\begin{array}{l}\text { Exclusively } \\
\text { breastfeeding }\end{array} \\
\mathrm{n}\end{array}$} & \multirow{2}{*}{$\begin{array}{l}\begin{array}{l}\text { Formula } \\
\text { feeding }\end{array} \\
\mathrm{n}\end{array}$} & \multicolumn{2}{|l|}{$\begin{array}{l}\text { Relation between contraception } \\
\text { and infant milk feeding }\end{array}$} \\
\hline & & & OR (95\% Cl) & \\
\hline 0-3 Months postpartum & & & $17.5(11.3-27.0)$ & $14.5(9.3-22.5)$ \\
\hline None or non-hormonal & 537 & 296 & & \\
\hline Hormonal & 25 & 241 & & \\
\hline Hormonal & 26 & 432 & & \\
\hline Total & 945 & 1423 & & \\
\hline
\end{tabular}

${ }^{1}$ Adjusted for maternal age, education, smoking and employment status.

\section{Censored data}

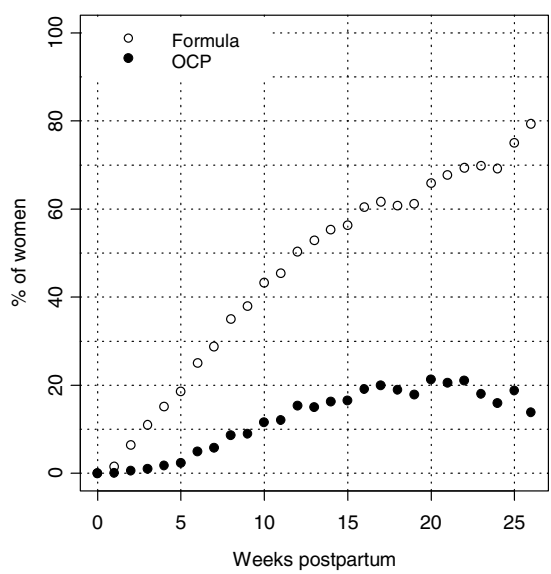

Imputed data

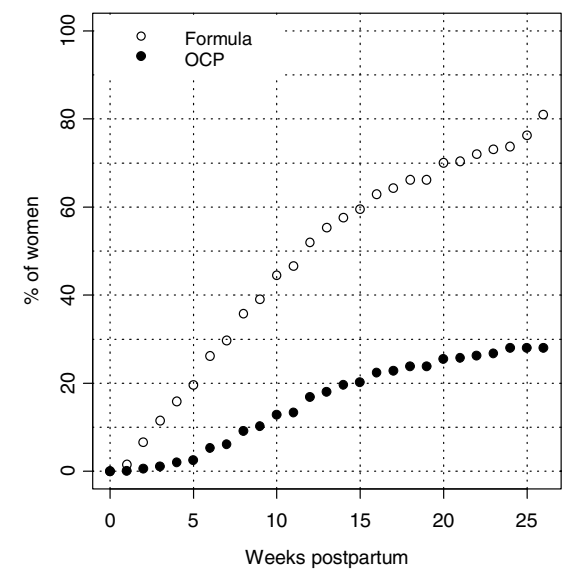

Figure 1 Percentages of women introducing formula and resuming $\mathrm{OCP}$, hormonal contraception after they started successful breastfeeding in the censored data, $(n=945)$ and for the imputed data $(n=1829)$.

status) 14.5 (9.3-22.5). During the second 3-month period (3-6 month), these odds ratios and 95\% confidence interval are, respectively, 13.1 (8.6-19.9) and 11.7 (7.6-17.9). We find that only 5\% of mothers who breastfed used hormonal contraception (mostly the $\mathrm{OCP}$ ), while this percentage was $47 \%$ in mothers who gave formula feeds. Equally, only $7 \%$ of the women who used hormonal contraception breastfed their infant. This percentage is 54\% for the women who used no or non-hormonal contraception (mostly condom).

The percentage of breastfeeding women that used the various types of contraception depended on the time after delivery. Figure 1 shows the percentage of women per week who introduced formula feeds and resumed the OCP in the raw censored data. Only women who successfully started breastfeeding were counted. Figure 1 also shows the increase of the percentage of women who started formula or hormonal contraception per week as calculated from the imputed data. Both estimation methods show that by the time $20-27 \%$ of all breastfeeding women have resumed the OCP (25 weeks postpartum) and $80 \%$ has introduced formula feeding.
There were 480 women who resumed taking the OCP and introduced formula before week 26 postpartum. Figure 2 illustrates the time lag between both events in this group. Irrespective of the infants' age, breastfeeding women introduced formula feeding before resuming hormonal contraception. Around week 6, 50\% had introduced formula feeds. Around week 12 , about $50 \%$ had started using the OCP. In fact, the most frequently observed time lag between introduction of formula milk and start of the OCP is 6 weeks.

Figure 3 shows the association between introduction of formula feeding and OCP resumption at the individual level. The points cluster above the diagonal, illustrating once again that most women resumed hormonal contraception (OCP) after the introduction of formula feeding.

\section{DISCUSSION}

This study addresses both infant milk feeding and practised contraception in a sizable population sample of 2710 Dutch mothers. We found a strong association between breastfeeding duration and contraception: mothers who breastfed 


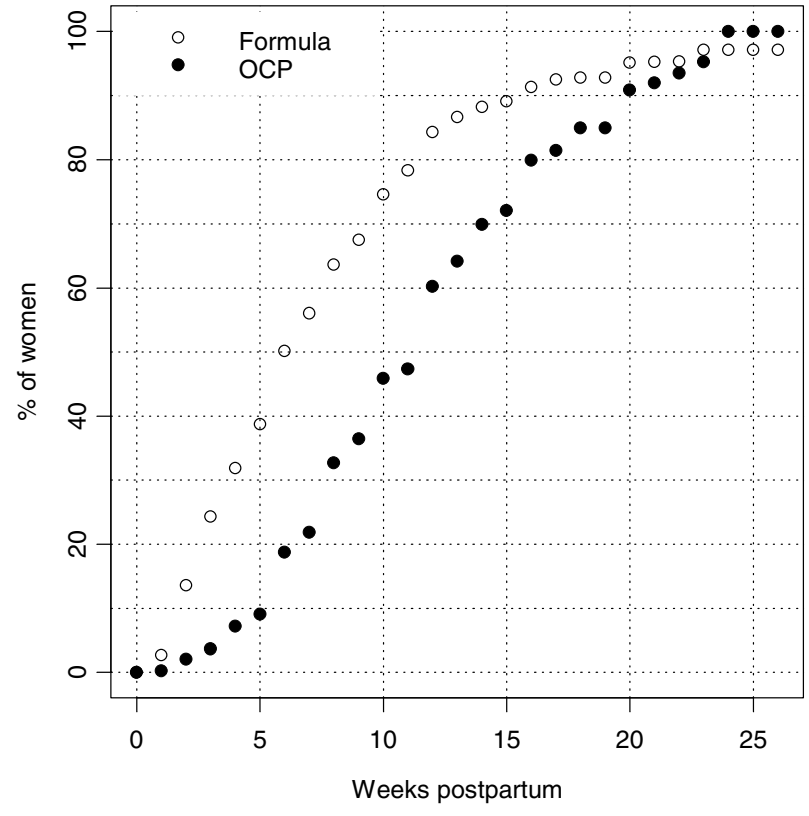

Figure 2 Percentages of women per week that introduced formula feeding and resumed $\mathrm{OCP}$, hormonal contraception (imputed data, $\mathrm{n}=480$ ).

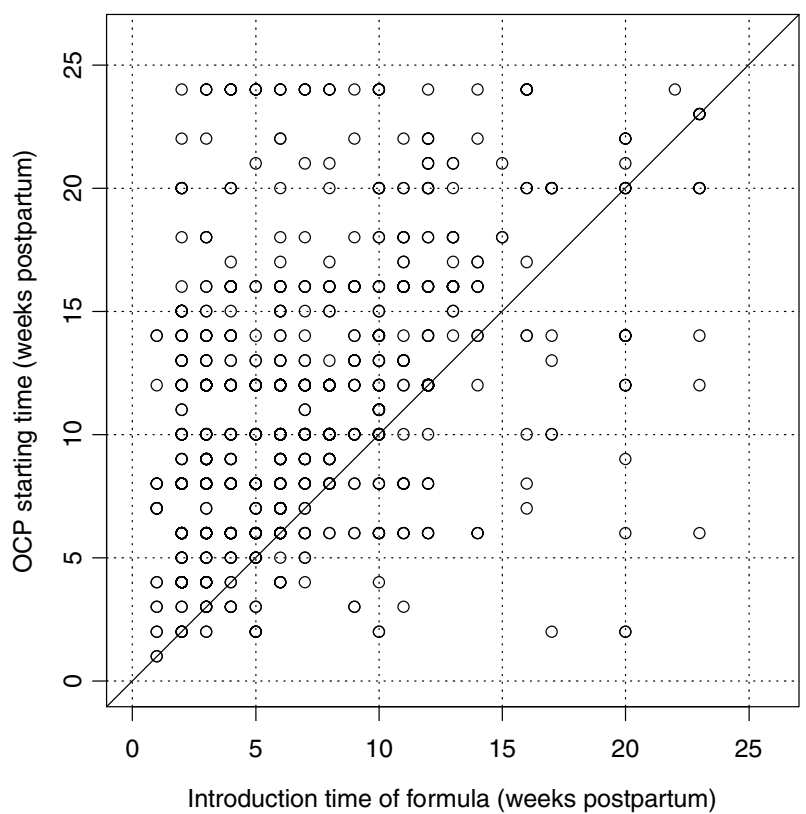

Figure 3 Scatter plot of the moment of resuming OCP, hormonal contraception (vertical axis) and the moment of introduction of formula feeding (horizontal axis) $(n=480)$. Since $88 \%$ of the points lie above the diagonal, most women resume OCP after the introduction of formula milk.

did not use hormonal contraception (the OCP), and mothers who took the OCP give formula feeds. Introduction of formula feeding precedes OCP resumption by 6 weeks.

One drawback of the study is that the self-reported data only permit tentative conclusions. We handed out a questionnaire to mothers up to 6 months postpartum. They recalled the infant feeding and contraceptive method, and the answers could be inaccurate. In order to investigate the magnitude of this problem, we conducted interviews among a similar group of 145 women at the child health clinics during a regular visit. The questionnaire was administered, and their personal opinions on the combination of various contraceptives with breastfeeding were registered. In these interviews, women expressed clear opinions on the combination of breastfeeding and OCP use, which were in agreement with the above analysis. About $77 \%$ of these interviewed mothers stated that the OCP should not be used during breastfeeding. They expressed fear for side effects of hormones present in their milk. Only $11 \%$ of them answered that they see no problem in combining the OCP and breastfeeding.

Another problem is that the study is not fully representative. Highly educated women are overrepresented in the study group. This may have had consequences for the reported frequencies of the several methods of contraception. In our analyses on the relation between contraception and breastfeeding, we made adjustments for, amongst others, maternal education. In addition, the strength of the association is so overwhelming that it is not likely that it is lost all together in a fully representative population.

The pattern of contraceptive methods used during the postpartum period clearly differs from the overall pattern in Dutch women. Only the proportion of IUD users (75 per 1000 Dutch women 25-45 years (12)) equals the 8\% we found during the second trimester postpartum. During both postpartum trimesters, fewer mothers used the OCP, compared to women of the same age in the general population $(22 \%$ vs. $40 \%)$. Condoms on the other hand were used more frequently (30\% vs. 9\%).

The main reason mentioned by Dutch mothers to stop breastfeeding is 'not enough breast milk' (3). We found that, on average, mothers introduce formula feeding 6 weeks prior to resuming hormonal contraception. So OCP use cannot be the cause to have to stop with breastfeeding. Other reasons, like inadequate or infrequent latching or general insecurity about their capability to satisfy their infant, must be ranked higher.

The effects of hormonal contraceptives on lactation and on the newborn have been reviewed systematically by King (13). It was concluded that there are insufficient data to establish any effect of OCP on milk quantity or quality (5). Furthermore, there is minimal evidence that exposure to hormones via human milk is harmful to the infant; however, there is no evidence to support complete safety. Until safety is guaranteed, the progesterone-only pill is the most suitable in combination with breastfeeding. Still, this is used infrequently in the Netherlands. The IUD during lactation is also a safe alternative to the widely used combined OCP $(14,15)$.

The interconnection between breastfeeding and contraceptive behaviour is relevant. WHO/UNICEF's Baby Friendly Hospital Initiative based on the 10 steps to successful breastfeeding has boosted the breastfeeding prevalence in the Netherlands and elsewhere (16). Contraceptive behaviour until now has not been a major subject in this context; however, we have shown that it is an 
important factor to consider. The choice of contraceptive method must be seen in conjunction with proper lactation care. In order to effectively promote longer duration of breastfeeding, health workers need to address lactation in the context of opinions on suitable contraceptive behaviour. It might even be considered to add the issue to the 10 steps to successful breastfeeding to promote longer duration of breastfeeding.

\section{ACKNOWLEDGEMENT}

The fieldwork of this study was supported by grant 2500.0001 from the Netherlands Organization for Health Research and Development (ZON-MW).

\section{CONFLICT OF INTEREST STATEMENT}

We declare that we have no conflict of interest.

\section{References}

1. Quigley MA, Kelly YJ, Sacker A. Breastfeeding and hospitalization for diarrheal and respiratory infection in the United Kingdom Millennium Cohort Study. Pediatrics 2007; 119: e837-42.

2. World Health Organization. The optimal duration of exclusive breastfeeding: report of an expert consultation. Geneva: WHO, 2001.

3. Lanting CI, Van Wouwe JP, Reijneveld SA. Infant milk feeding practices in the Netherlands and associated factors. Acta Paediatrica 2005; 94: 935-42.

4. World Health Organization. Improving access to quality care in family planning. Medical eligibility criteria for contraceptive use. 3rd ed. Geneva: WHO, 2004.

5. Truitt ST, Fraser A, Gallo MF, Lopez LM, Grimes DA, Schulz KF. Combined hormonal versus nonhormonal versus progestin-only contraception in lactation (Review). Cochrane Database Syst Rev 2003; CD003988.

6. Glasier A, Brechin S, Raine R, Penney G. A consensus process to adapt the World Health Organization selected practice recommendations for UK use. Contraception 2003; 68: 327-33.

7. Boukes FS, Beijderwellen L, Van Der Does en FEE, Assendelft WJJ. Samenvatting van de standaard 'Hormonale anticonceptie' (tweede herziening) van het Nederlands Huisartsen Genootschap [Summary of the practice guideline 'Hormonal contraception' (second revision) from the Dutch College of General Practitioners]. Ned Tijdschr Geneeskd 2004; 148: 1285-9.

8. Treffers PE. Borstvoeding en anticonceptie [Breastfeeding and contraception]. Ned Tijdschr Geneeskd 1999; 143: 1900-4.

9. Rice S, Coombs D, Fish L, Leeper J. Breast-feeding and contraception in Peru. J Health Popul Nutr 2002; 20: 51-8.

10. Van Buuren S, Boshuizen HC, Knook DL. Multiple imputation of missing blood pressure covariates in survival analysis. Stat Med 1999; 18: 681-94.

11. Van Buuren S. Multiple imputation of discrete and continuous data by fully conditional specification. Stat Methods Med Res 2007; 16: 219-242.

12. University Medical Centre St Radboud Nijmegen. Continuous Registration of Morbidity Nijmegen 1999-2003 (in Dutch). 2004.

13. King J. Contraception and lactation. J Midwifery Womens Health 2007; 52: 614-20.

14. WHO Task force for Epidemiological Research on Reproductive Health. Progestin-only contraceptives during lactation: I. Infant growth. Contraception 1994; 50: 35-53.

15. WHO Task force for epidemiological Research on Reproductive Health. Progestin-only contraception during lactation: II. Infant development. Contraception 1994; 50: 55-68.

16. Merten S, Dratva J, Ackermann-Liebrich U. Do baby-friendly hospitals influence breastfeeding duration on a national level? Pediatrics 2005; 116: e702-8. 